\title{
Code Park: A New 3D Code Visualization Tool
}

\author{
Pooya Khaloo*, Mehran Maghoumi ${ }^{\dagger}$, Eugene Taranta II ${ }^{\ddagger}$, David Bettner ${ }^{\S}$, Joseph Laviola Jr. ${ }^{\text {I }}$ \\ University Of Central Florida \\ Email: * pooya@cs.ucf.edu, †mehran@cs.ucf.edu, ${ }^{\star}$ etaranta@gmail.com, ${ }^{\S}$ dbettner@gmail.com, ${ }^{\dagger}$ jjl@ cs.ucf.edu
}

\begin{abstract}
We introduce Code Park, a novel tool for visualizing codebases in a 3D game-like environment. Code Park aims to improve a programmer's understanding of an existing codebase in a manner that is both engaging and intuitive, appealing to novice users such as students. It achieves these goals by laying out the codebase in a 3D park-like environment. Each class in the codebase is represented as a 3D room-like structure. Constituent parts of the class (variable, member functions, etc.) are laid out on the walls, resembling a syntax-aware "wallpaper". The users can interact with the codebase using an overview, and a firstperson viewer mode. We conducted two user studies to evaluate Code Park's usability and suitability for organizing an existing project. Our results indicate that Code Park is easy to get familiar with and significantly helps in code understanding compared to a traditional IDE. Further, the users unanimously believed that Code Park was a fun tool to work with.
\end{abstract}

\section{INTRODUCTION}

Code visualization techniques assist developers in gaining insights into a codebase that may otherwise be difficult or impossible to acquire via the use of a traditional text editor. Over the years, various techniques have been proposed to address different issues. For instance, SeeSoft [1] maps each line of code to an auxiliary UI bar such that the color of the bar represents a property of the code, e.g. red rows could represent recently changed lines. Code Bubbles [2] organizes source into interrelated editable fragments illustrated as bubbles within the user interface, and CodeCity [3] uses a 3D city-like structure to visualize the relative complexity of modules within a codebase. However, among all of the techniques that have been explored, little attention has been given to how source code can be visualized in order to help developers become familiar with and learn a new codebase.

The task of learning a new codebase is fraught with many issues, three of which include memorization, cognitive load, and engagement [4]-[6]. Imagine a new hire set to study a proprietary project that is a complex hierarchical conglomeration of thousands of source files. Indeed memorizing the structure of such a system is a difficult chore, not to mention mentally exhausting as well as potentially tedious and likely boring. To address these issues, we introduce Code Park (CP), a new 3D code visualization tool that aims to improve a programmer's understanding of an existing codebase in a manner that is both engaging and fun. $\mathrm{CP}$ organizes source code into a 3D scene in order to take advantage of human's spatial memory capabilities [7] and help one better understand and remember the architecture. $\mathrm{CP}$ also supports two points of view that are an exo-centric (bird's eye) and ego-centric (first-person) view, which allows one to examine the codebase at different granularities (see Figure 11).

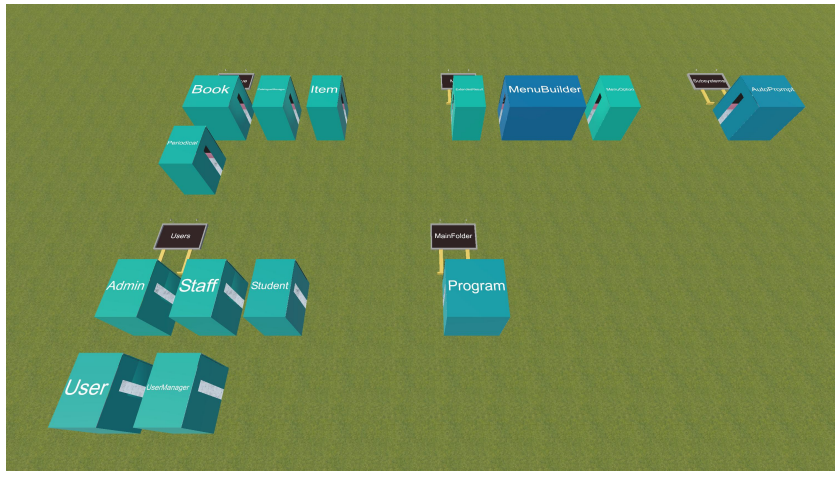

(a) Top-down view of the entire codebase. Each cube is a code room.

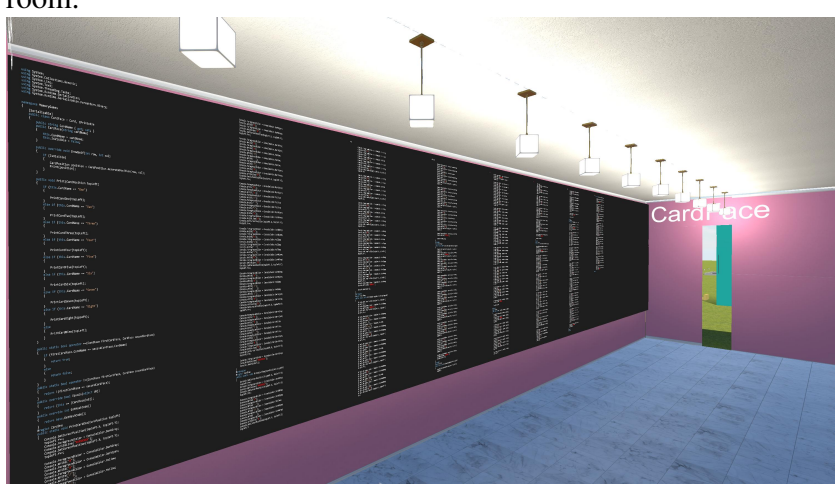

(b) Exploring the inside of a code room in first-person vieweing mode.

Fig. 1: Code Park in action. Each class is shown as a room. The user can study the code in a first-person view mode.

Our design goals with CP are threefold. We aimed to create a code visualization tool that helps users become familiar with and memorizing an existing codebase. This tool must be easy to work with and must show information in a form which reduces the user's cognitive load. Finally, the tool must be engaging and enjoyable to work with. Specifically the following are the contributions of our exploratory work:

1) Creating an engaging and intuitive tool for improved code understanding.

2) Examining the usability of such a tool and gauging user interest.

3) Displaying the source code itself in a 3D environment (rather than a metaphorical representation) and facilitate "intimate" interaction with the code via an ego-centric mode.

To evaluate our design goals and the usability of the 
system, we performed a user study. Our results indicate that our participants found CP easy to use and helpful in code understanding. Additionally, they unanimously believed that $\mathrm{CP}$ was enjoyable. We ran a follow up user study to determine how users would organize the classes of an existing project in the $3 \mathrm{D}$ environment in a way that the arrangement helps them remember the code structure better.

\section{RELATED WORK}

There is a body of work available in the literature on software visualization $[8]-[14]$. We direct the reader to comprehensive surveys of different methods available in the work of Teyseyre and Campo [15] and also Caserta and Olivier [16]. For example SeeSoft [1], one of the earliest visualization metaphors, allows one to analyze up to 50,000 lines of code by mapping each line of code into a thin row. Marcus et al. [17] added a new dimension to SeeSoft to support an abstraction mechanism to achieve better representation of higher dimensional data.

Recently, there have been more work focusing on $2 \mathrm{D}$ visualization. Code Bubbles [2] suggested a collection of editable fragments that represent functions in a class. Code Gestalt [18] used tag overlay and thematic releations. Lanza and Ducasse [19] proposed categorizing classes and their internal objects into blocks called Blue Prints. Gutwenger et al. [20] proposed an approach for improved aesthetic properties of UML diagrams when visualizing hierarchical and nonhierarchical relations. Balzer et al. $[21]$ introduced hierarchybased visualization for software metrics using Voroni Treemaps. Additionally, Holten [22] used both hierarchical and nonhierarchical data to visualize adjacency relation in software. The common observation among these efforts is that they are all based on 2D environments and were mostly suitable for expert users.

Benefit of 3D over 2D. Remembering code structure will result in faster development so it is an essential part of being a programmer. Specifically, 3D environments tap into the spatial memory of the user and help with memorizing the position of objects [7]. These objects could be classes or methods. There are also studies which provide evidence that spatial aptitude is a strong predictor of performance with computer-based user interfaces. For instance, Cockburn and McKenzie [23] have shown that 3D interfaces that leverage the human's spatial memory result in better performance even though some of their subjects believed that 3D interfaces are less efficient. Robertson et al. [24] have also shown that spatial memory does in fact play a role in $3 \mathrm{D}$ virtual environments. A number of researchers have attempted to solve the problem of understanding code structure. Graham et al. [25] suggested a solar system metaphor, in which each planet represented a Java class and the orbits showed various inheritance levels. Balzer et al. [26] presented the static structure and the relation of object-oriented programs using 3D blocks in a 2D landscape model.

Among these, the city metaphor is one of the most popular ideas. CodeCity [3] is an example of a city metaphor. CodeCity focus on the visualization of large and complex codebases using city structures where the number of methods in each class represented the width of the buildings and the number of attributes represented their height. Panas et al. [27] used Vizz3D1 10 visualize communication between stakeholders and developers in the process of software implementation. Alam and Dugerdil [28] introduced the Evospaces visualization tool in which they represent files and classes with buildings similar to CodeCity. Additionally, they showed the relations between the classes using solid pipes. There are other works that leverage such visualization metaphors such as [29] and [30]. The common theme with these tools is that most of them only showed the name of the classes in their environment which is not instrumental for learning purposes and again, they are mainly targeting experienced developers.

User engagement. The fun and engaging aspect of programming tools, especially for teaching purposes, has gained attention in recent years. Alice [31] is a 3D interactive animation environment tool that aims to encourage student engagement by creating a game-like environment. Resnik et al. [32] introduced a novel development environment that appeals to people who believe their skills are not on par with experienced developers. They designed their tool in a way that was fun and likable. They also made it suitable for youth learners. There are also many studies that are focused on creating fun and engaging tools and methods that help newcomers enjoy learning programming [33]-[36].

What sets CP apart from current tools in the literature is that it is designed to be suitable for both beginner and experienced developers alike. Saito et al. [37] examined the learning effects between a visual and a text-based environment on teaching programming to beginners. Their results deemed the visual environment as a more suitable option for teaching beginners. Indeed, at the lines of code level, CP differs little from a traditional IDE, other than code can be read from within a $3 \mathrm{D}$ environment. Where CP diverges from a traditional IDE is in how one interacts with a project: file hierarchies vs buildings in 3-space. The exo-centric view in CP helps the user glean a holistic understanding of the codebase without involving them in unnecessary details. Conversely, the ego-centric view enables a fine-grained understanding of the codebase. The addition of the ego-centric view is the key distinction between the current work and CodeCity [3].

\section{USER INTERFACE DESIGN}

When designing $\mathrm{CP}$, we had three main goals in mind. We wanted CP to greatly help with learning codebases, be easy to learn and fun to use. With these goals in mind, we employed an iterative design approach. We tested $\mathrm{CP}$ often and refined it by incorporating peer feedback from experts in this area.

In CP each room represents one class in the codebase. This approach is inspired by CodeCity [3]. The rooms are placed on a ground filled with a grassy texture, which resembles a park. The rooms are grouped together based on the code's project file structure: files inside the same directory result in adjacent rooms and each group of rooms are labeled by the

\footnotetext{
${ }^{1}$ http://vizz3d.sourceforge.net/
} 


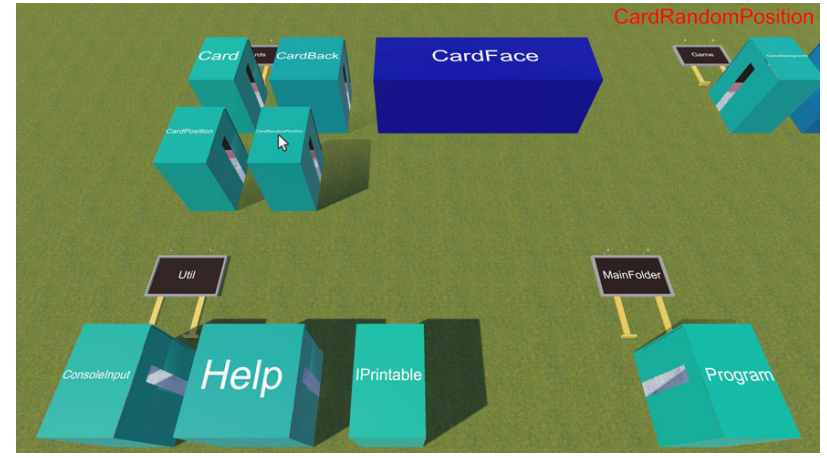

(a) Bird's view with tooltip on top-right.

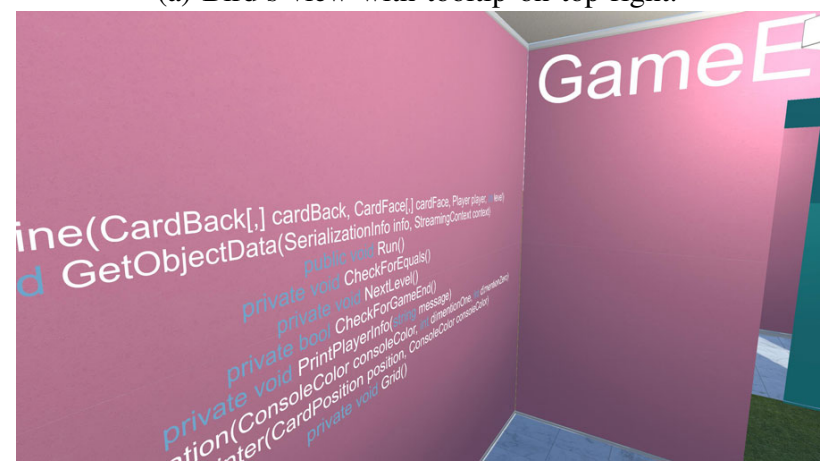

(c) Class method overview wallpaper.

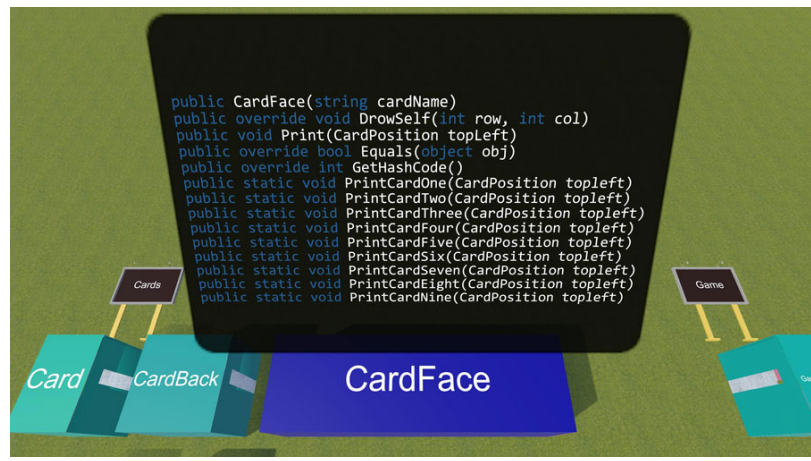

(b) Class method overview tooltip.

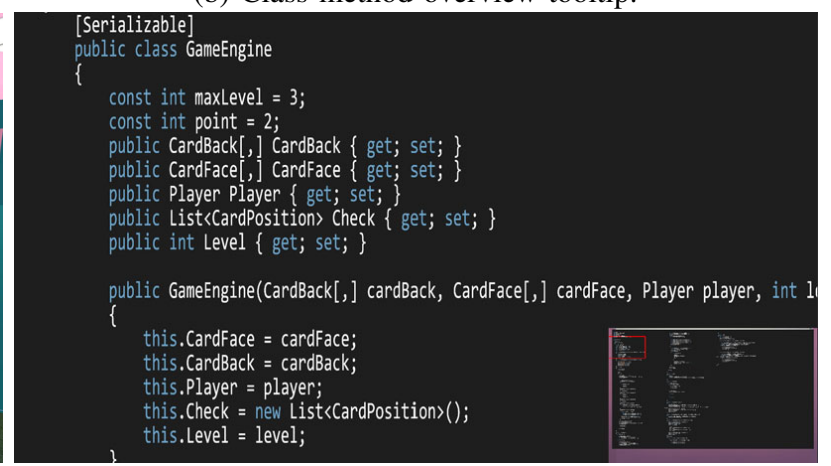

(d) Code reading view.

Fig. 2: Various CP view modes and features. In (a), the user has hovered the mouse over a class with a long name.

directory name (see Figure 2a). This was done in order to avoid confusion for large codebases that had many files. The size and the color of each room is proportional to the size of the class they contain (larger classes appear as larger rooms that have a darker color on their exteriors). Each room has syntax-aware wallpapers that have a color theme matching Microsoft Visual Studio (VS)'s dark theme (dark background and gray text with colored language keywords, comments and strings literals - see Figure 2d, The users can explore the environment in the first-person mode because it is one of the well known navigation methods in the $3 \mathrm{D}$ environment and also resembles video games which helps making $\mathrm{CP}$ more engaging. The users can click on the wallpaper with the cross-hair to transition into the code viewing mode. This places the camera orthogonal to the wallpaper, allowing the users to read the code and scroll it (using the mouse wheel) similar to a text editor.

One of our design goals was to allow CP to show as much information as possible while maintaining the ease of use and managing the user's cognitive load. Consequently, we employed the bird's view navigation mode (see Figure 2a). In bird view mode, the users have a top-down view of all the rooms placed on the ground. This way, the users can first get some insight about the structure of the codebase and the number of classes and then study the codebase in more detail via the first-person mode. In bird's view, the name of each class is etched on the roof of each room. This immediately helps the users know which class they are looking at. In this view, hovering the cursor over each room shows a tool-tip text showing the name of the class in a larger font as shown in Figure 2a. The users can transition to the first-person view by clicking one of the code rooms and can go back to bird's view by pressing a keyboard shortcut. The camera's transition to and from bird's view is animated in order to preserve the user's sense of spatial awareness.

We improved bird's view mode further by adding syntax parsing features so as to be able to provide class-level details (such as the list of all member functions) to the user. In bird's view, right-clicking on a room shows a tool-tip balloon that provides a list of the methods defined in the class corresponding to the room (see Figure 2b). This allows the users to quickly glean useful information about each class. This overview is also available on one of the wallpapers inside each room as shown in Figure 2c.

By supporting syntax parsing, we implemented a commonly used feature in most IDEs, namely go-to definition. This feature allows the programmers to quickly jump to the location inside a file where a user-defined type or a variable is defined for the first time. It aids the users in learning the codebase by allowing them to both mentally and visually connect the disparate parts of the code together.

In $\mathrm{CP}$, the users can click on a variable, function or a userdefined type with their cross-hair and jump to the location where the clicked item is defined for the first time. The jump from a room to another room is done using two consecutive animated camera transitions: from the current room to bird's 
view and from bird's view to the room containing the definition ${ }^{2}$ After the transition is finished, the definition of interest is signified by a blinking highlight to focus the user's attention and also to indicate the completion of the task. In addition to being visually appealing, these transitions maintain the users awareness of the $3 \mathrm{D}$ environment. It is worth mentioning that all movements and navigation actions in $\mathrm{CP}$ are done with animations instead of jumping around which helps preserve the spatial awareness of users. This way they can memorize location of each part of codebase such as classes or even methods and variables more efficiently.

\section{EVALUATION: CODEPARK USABILITY}

We conducted a usability study to evaluate the following hypotheses:

H1: A project organized in a 3-space, city-like environment will be easier to learn.

H2: A project organized in a game-like environment will be more engaging.

H3: The move to 3-space will not make working with source more difficult.

At the time of this writing, CP only supports $\mathrm{C \#}$ projects. Therefore, we decided to compare $\mathrm{CP}$ with one of the most prominently used IDEs for C\#, namely Microsoft Visual Studio (VS). Given a few programming-related tasks, we are interested in studying the effects that using $\mathrm{CP}$ has and also determine how CP helps with code understanding.

Participants and Equipment. We recruited 28 participants from University of Central Florida (22 males and 6 females ranging in age from 18 to 31 with a mean age of 22.8). Our requirements for participants were that they should be familiar with the C\# language and also have prior experience with VS. All participants were compensated with $\$ 10$ for their efforts. Each participant was given a pre-questionnaire containing some demographic questions as well as some questions asking about their experience in developing $\mathrm{C \#}$ applications. After that, a short C\# skill test was administered to validate their responses in the pre-questionnaire. The skill test contained five multiplechoice questions with varying difficulties selected from a pool of coding interview questions 3

After the skill test, each participant was given a set of tasks to perform using each tool (VS or CP) and filled out postquestionnaires detailing their experience with each tool. Prior to performing the tasks on a specific tool, the participants were given a quick tutorial of both tools and were given a few minutes to warm up with the tool.

At the end of the study, the participants were asked to fill out a post-study questionnaire to share their overall experience. The duration of the user study ranged from 60 minutes to 90 minutes depending on how fast each participants progressed towards their assigned tasks.

Our setup consisted of a 50-inch Sony Bravia TV used as the computer screen. The users used Visual Studio 2013

\footnotetext{
${ }^{2}$ Unless the definition is inside the same room in which case the camera is transitioned to the definition directly.

$\sqrt[3]{\text { https://www.interviewmocha.com/tests/c-sharp-coding-test-basic }}$
}

Community Edition and Unity3D v5.4.0 on a machine running Microsoft ${ }^{\circledR}$ Windows 10 64-bit equipped with 16.0 GB of RAM, Intel $^{\circledR}$ Core $^{\mathrm{TM}}$ i7-4790 processor with 4 cores running at 3.60 $\mathrm{GHz}$ and NVIDIA GeForce GTX 970 graphics processor.

Experiment Design and Procedure. When comparing VS with $\mathrm{CP}$, there are a few considerations involved in order to design a sound experiment that allows a fair comparison between the two tools. First, VS has been in development for many years and most $\mathrm{C \#}$ developers work with VS frequently. As a result, it could be the case that the users who use VS frequently are biased towards VS, because they have had more time to work and get comfortable with it. Consequently, devising a fair between-subject design would be difficult.

Second, focusing on a purely within-subject design presents other complications. It is important to avoid any unwanted learning effects in a within-subject design when the user is working with both tools. Given a particular codebase and a set of tasks, if a user performs those tasks in VS and then switches to CP to perform the same set of tasks, chances are that those tasks are performed much faster the second time. This is because the user will have learned the codebase's structure the first time and can leverage that knowledge in $\mathrm{CP}$. To avoid this learning effect, the user should be given two different codebases to work with. However, care must be taken when selecting the two codebases, as they should be relatively similar in structure and the degree of difficulty.

Having two codebases may pose another problem. Even if the two codebases are specifically chosen to be similar, minor differences between the two could affect the results. Moreover, studying and learning someone else's code can quickly become tedious and the users can become fatigued after using the first tool, affecting their performance in the second tool. Therefore, it is imperative to mitigate any of these unwanted effects in the study.

Facing with all these considerations, we opted to use a mixed-effects design for our study to benefit from both of the design modes. In our experiments, each participant used two different codebases with both tools. Several codebases were considered at first and after a few pilot runs and getting feedback from peers, two codebases were carefully selected. These selected codebases shared similar structures and properties. One codebase is a console-based library catalog system called Library Manager (LM). The other codebase is a console-based card matching game called Memory Game (MG). Table I] summarizes these codebases. Note that even though MG contains more lines of code, some of its largest classes contain mostly duplicate code that handle drawing the shape of each playing card on the console window. Our assumption with the choice of codebases is that the two codebases are not significantly different and would not bias our results 4

To avoid the unwanted effects discussed previously, we permute the order of tools and codebases across participants. As a result, each participant started the experiment with either VS or CP. Also their first tasks were performed on either LM

\footnotetext{
${ }^{4}$ This assumption will later be examined in the Discussion section.
} 
TABLE I: Comparison of the two codebases used in the user studies: Library Manger (LM) and Memory Game (MG). LoC is lines of code reported by the line counting program cloc $^{5}$

\begin{tabular}{cccc}
\hline Codebase & No. Classes & LoC & LoC (largest class) \\
\hline LM & 14 & 977 & 237 \\
MG & 16 & 1753 & 791 \\
\hline
\end{tabular}

TABLE II: Different experiment groups. Group names are only for tracking the number of participants in each permutation of the study.

\begin{tabular}{ccccc}
\hline $\begin{array}{c}\text { Group } \\
\text { Name }\end{array}$ & $\begin{array}{c}\text { First } \\
\text { Tool }\end{array}$ & $\begin{array}{c}\text { First } \\
\text { Codebase }\end{array}$ & $\begin{array}{c}\text { Second } \\
\text { Tool }\end{array}$ & $\begin{array}{c}\text { Second } \\
\text { Codebase }\end{array}$ \\
\hline A & VS & LM & CP & MG \\
B & CP & MG & VS & LM \\
C & VS & MG & CP & LM \\
D & CP & LM & VS & MG \\
\hline
\end{tabular}

or MG. The possible permutations divided our participants into four groups detailed in Table II By recruiting 28 participants, we had 7 participants per group. We randomly assigned a participant to a group. This results in a balanced mixed-effects design in which all possibilities and orders are considered.

On each codebase, the participants were asked to perform five tasks, each with a varying degree of difficulty. These tasks are presented in Table III Among these, some tasks force the participant to explore the codebase, whereas other tasks were directly related to a participant's understanding of the codebase, the program structure and the logic behind it. Tasks T1 and T2 were similar for both codebases. Task T3 asked about the object-oriented relationship between classes $A$ and $B$. In LM, this relationship was inheritance and in MG this relationship was having a common parent class ( $A$ and $B$ are siblings $\sqrt{6}$ Prior to being tasked with $\mathrm{T} 4$, the participants were asked to try out a particular feature of the program. Upon the trial, the program would crash prematurely and no output was produced. The participants were told that the reason for this behavior was a simple intentional bug and were tasked with finding the likely location of the bug. For task T5, the participants were asked to imagine a scenario where somebody asked them about their approach for adding a new feature to the particular codebase that they were working on. In LM, they were asked to add additional menu options for specific types of users. In MG, they were asked to modify the scoring system to penalize the player for each mistake. We should note that neither of these tasks involve writing any code. This was necessary because at the time of this writing, CP did not incorporate a code editor. Also, we are primarily interested in determining the effects of CP on code understanding. The participants were responsible for showing a suitable location in the logic to add these features. There were multiple valid locations for each task in either codebase and the participants were free to select any of the valid locations. When performing any of these tasks,

\footnotetext{
5 http://cloc.sourceforge.net/

${ }^{6}$ The selected classes were the same for all participants.
}

TABLE III: Participant tasks. Each task was timed. We used these measurements for our quantitative analysis.

\begin{tabular}{ll}
\hline & \multicolumn{1}{c}{ Task } \\
\hline T1 & Find a valid username to login into the program. \\
T2 & Find an abstract class in the codebase. \\
T3 & Determine the relationship between classes $A$ and $B$. \\
T4 & Find an intentional bug that causes a program crash. \\
T5 & Pinpoint a reasonable location in the code for adding the \\
& necessary logic to support feature $X$. \\
\hline
\end{tabular}

TABLE IV: Post-task questionnaire. Participants answered these questions on a 7-point Likert scale after finishing their tasks with both tools. We used this data for our qualitative analysis.

\begin{tabular}{l} 
Post Task Questionnaire \\
\hline Q1 I found it easy to work with CP/VS. \\
Q2 I found it easy to become familiar with CP/VS. \\
Q3 CP/VS helps me become familiar with codebase's structure. \\
Q4 It was easy to navigate through the code with CP/VS. \\
Q5 It was easy to find the definition of some variable with CP/VS. \\
Q6 How much did you like CP/VS? \\
Q7 How did you feel when using the tool? \\
Q8 It was easy to find what I wanted in the code using CP/VS.
\end{tabular}

the users were explicitly told that they were not allowed to use the debugging features of VS, nor the search functionality for finding a specific type or class. They were, however, permitted to use VS's built-in go-to definition feature by holding down the control key and clicking with the mouse on a user-defined type. After performing the tasks, the participants were given three questionnaires to fill out.

Metrics. For quantitative data, we recorded the time each participant took to perform each task. The qualitative measurement was performed using the post-task and post-study questionnaires. After performing the tasks with each tool, the participants were given a post-task questionnaire that asked them to share their experience about the tool they worked with. These questions were the same for both tools and are shown in Table IV. The responses to these questions were measured on a 7 -point Likert scale $(1=$ the most negative response, $7=$ the most positive response).

Upon the completion of all tasks, the participants were given a post-study questionnaire to measure their preferences of both tools from different aspects. This questionnaire is detailed in Table V] The participants were required to select either VS or $\mathrm{CP}$ in their responses to each question.

\section{A. Results}

As mentioned before, we recorded quantitative as well as qualitative data. To analyze these data, we divided all of our participants into two equally sized groups based on their experience in $\mathrm{C \#}$ and software development. We leveraged the results of the $\mathrm{C \#}$ skill-test as well as the self-declared answers to determine the skill level of each participants. The skill-test questions were weighted twice as much in order to 
TABLE V: Post-study questionnaire. Participants answered these questions after finishing all their tasks with both tools. The answer to each question is either CP or VS. We used this data for qualitative analysis.

\begin{tabular}{cl}
\hline & \multicolumn{1}{c}{ Post Study Questionnaire } \\
\hline SQ1 & Which tool is more comfortable to use? \\
SQ2 & Which tool is more likable? \\
SQ3 & Which tool is more natural? \\
SQ4 & Which tool is easier to use? \\
SQ5 & Which tool is more fun to use? \\
SQ6 & Which tool is more frustrating? \\
SQ7 & Which tool helps you more in remembering the \\
& codebase structure? \\
SQ8 & Which tool do you prefer for learning a codebase? \\
SQ9 & Which tool do you prefer for a codebase \\
& you are already familiar with for additional work? \\
SQ10 & Which tool do you prefer for finding a particular \\
& class/variable? \\
SQ11 & Which tool do you prefer for tracking down a bug? \\
SQ12 & Overall, which tool is better?
\end{tabular}

reduce potential over- or undervaluation of the self-declared responses.

In summary, our experiments have three factors: tool, codebase and experience. The tool factor has two levels: CP or VS, the codebase factor has two levels: LM or MG and the experience factor has two levels: beginner or expert.

1) Quantitative Results: Our quantitative results are based on the time a participant took to complete an assigned task. When validating ANOVA assumptions, we found that most group response variables failed the Shapiro-Wilk normality tests. Since our factorial design contains 3 factors (Codebase $\times$ Tool $\times$ Experience) with two levels each, we decided to utilize the Aligned Rank Transform (ART) [38] to make the data suitable for ANOVA. The mean time task completion results could be find in Figure 3 The analysis of these results are presented in Table VI.

2) Qualitative Results: Our qualitative results are comprised of two parts. The first part consists of the responses of the participants to the Likert-scale questions in the post-task questionnaire. The second part consists of the responses of the participants to the questions in the post-study questionnaire.

a) Post-Task Questionnaire: The responses to the Likertscale questions in our post-task questionnaire failed the ShapiroWilk normality tests. As such, similar to our quantitative results, we utilized ART [38] for ANOVA. Table VII] summarizes the analyses of our data. Average ratings for the eight post-task questions are shown in Figure 4

b) Post-Study Questionnaire: The post-study questionnaire required the participant to pick one tool for each question (see Table V). Since each question had only two choices, we used Chi-squared test to analyze the results. Table VIII summarizes the analyses of our data. We noticed that a few participants left some of the questions unanswered.

\footnotetext{
${ }^{7}$ All error bars are standard error values.
}

\section{B. Discussion}

The goal of this user study was to determine the degree to which we achieved our design goals with CP. Specifically, we were interested in determining how much it helps in learning a codebase (H1), how engaging is to work with $\mathrm{CP}$ especially for novice users (H2), how the users feel about working with it and how it compares against a traditional IDE such as VS (H3). Our results can be discussed from various aspects.

On the tool level and from a qualitative aspect, it is evident that the participants found CP significantly easier than VS to get familiar with, even though all participants had prior familiarity and experience with VS. The participants also found $\mathrm{CP}$ to be significantly more beneficial in becoming familiar with a codebase compared to VS. Both of these results were obtained regardless of the participant's experience, i.e. both experts and beginners found CP to be superior than VS in these two categories and this confirm our first and third hypotheses. Referring to the post-study questionnaire (see Table V), we see that the participants believed CP to be more likable compared to VS. Further, they believed that CP not only helped in learning the code structure but also helped them in remembering the code they studied and also finding the code elements that they were looking for. The interesting observation here is that all 28 participants unanimously believed that CP was more enjoyable which indicates that we achieved our goal of designing an engaging tool $(\mathrm{H} 2)$. Note that the results in all these categories were statistically significant. These results are further corroborated by the written feedback that our participants provided. Participants found CP to be "generally easier [than VS] to understand the structure of the code" and also felt that they were "using [their] spatial memory" when working with CP. These results show that regardless of the participant's experience our three hypotheses are valid.

In the remaining qualitative categories, there was no significant difference observed in users' responses between $\mathrm{CP}$ and VS. This is again interesting because VS has been in development since 1997 and has gone through many iterations and refinements, whereas $\mathrm{CP}$ has only been in development for about 6 months.

Our results indicate an interaction between the tool's ease of use and the participants' experience levels (Q1). A closer look at the responses (see Figure 5a) reveals that the beginners found $\mathrm{CP}$ to be easier to work with. Conversely, experts found VS to be easier. This coincides with what one would expect: the prior experience of the experts with VS gives them an edge in performing the assigned task. As a result, those participants found VS easier to work with compared to CP. When asked about their opinion, one user described CP as "an amusement park for beginners". Another user told us: "With more polish (smoother experience), I think there is promise for CP (especially for new programmers)".

We observe an interaction effect between tool and the codebase for users' perceived facility with finding variable definitions (Q5). The mean responses for this question are detailed for each codebase in Figure 5b. From LM to MG, we see an increase in the mean response values for $\mathrm{CP}$, but 


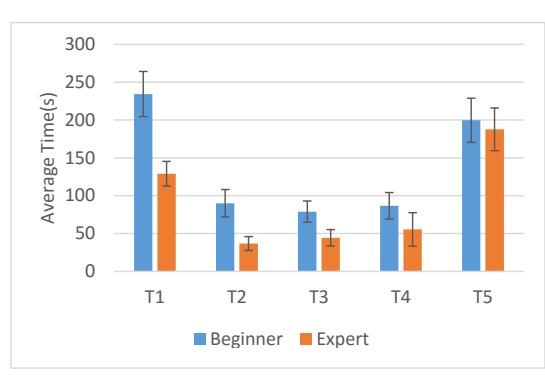

(a) MTTC by experience level.

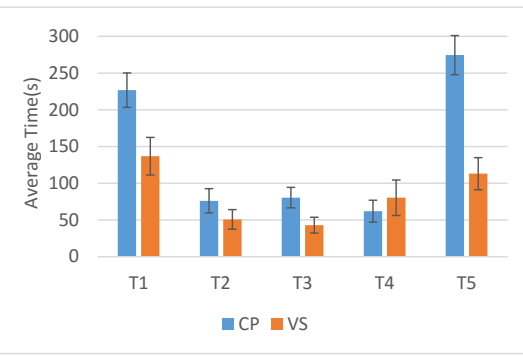

(b) MTTC based on the tool used.

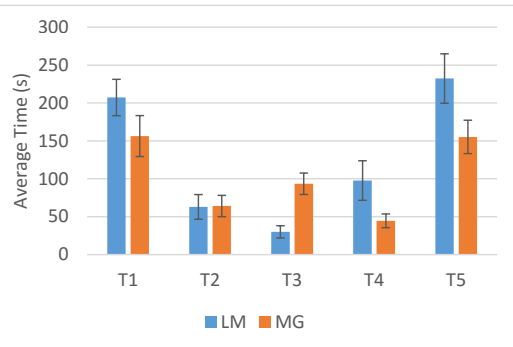

(c) MTTC based on the codebase.

Fig. 3: Mean time to task completion (MTTC). All time values are reported in seconds.

TABLE VI: ANOVA results on the quantitative data (time to task completion). Statistically significant results (95\% confidence) are highlighted in gray. The first column (TT) is task time. Codebase is abbreviated as $C B$. Experience level is abbreviated as Exp.

\begin{tabular}{|c|c|c|c|c|c|c|c|c|c|c|c|c|c|c|}
\hline \multirow{2}{*}{ TT } & \multicolumn{2}{|c|}{ CB } & \multicolumn{2}{|c|}{ Tool } & \multicolumn{2}{|c|}{ Exp. } & \multicolumn{2}{|c|}{ Tool $\times$ CB } & \multicolumn{2}{|c|}{ CB $\times$ Exp. } & \multicolumn{2}{|c|}{ Tool $\times$ Exp. } & \multicolumn{2}{|c|}{ Tool $\times$ CB $\times$ Exp. } \\
\hline & $F_{1,24}$ & $p$-value & $F_{1,24}$ & $p$-value & $F_{1,24}$ & $p$-value & $F_{1,24}$ & $p$-value & $F_{1,24}$ & $p$-value & $F_{1,24}$ & $p$-value & $F_{1,24}$ & $p$-value \\
\hline T1 & 3.92 & $=0.06$ & 12.0 & $<0.05$ & 7.53 & $<0.05$ & 0.78 & $=0.39$ & 0.49 & $=0.49$ & 0.19 & $=0.66$ & 0.10 & $=0.75$ \\
\hline $\mathbf{T} 2$ & 1.18 & $=0.29$ & 0.32 & $=0.58$ & 3.71 & $=0.07$ & 0.46 & $=0.50$ & 0.63 & $=0.44$ & 0.04 & $=0.84$ & 0.46 & $=0.51$ \\
\hline T3 & 45.2 & $<0.05$ & 15.9 & $<0.05$ & 5.70 & $<0.05$ & 1.02 & $=0.32$ & 0.58 & $=0.46$ & 6.01 & $<0.05$ & 0.00 & $=0.95$ \\
\hline T4 & 0.70 & $=0.41$ & 0.03 & $=0.86$ & 3.56 & $=0.07$ & 0.15 & $=0.70$ & 0.11 & $=0.74$ & 0.10 & $=0.76$ & 0.71 & $=0.41$ \\
\hline T5 & 7.71 & $<0.05$ & 41.9 & $<0.05$ & 0.08 & $=0.78$ & 3.93 & $=0.06$ & 1.35 & $=0.26$ & 0.32 & $=0.58$ & 0.05 & $=0.82$ \\
\hline
\end{tabular}

TABLE VII: ANOVA results on the qualitative data (post-task questionnaire). Statistically significant results (95\% confidence) are highlighted in gray. Codebase is abbreviated as $C B$. Experience level is abbreviated as Exp.

\begin{tabular}{|c|c|c|c|c|c|c|c|c|c|c|c|c|c|c|}
\hline \multirow{2}{*}{ Question } & \multicolumn{2}{|c|}{ CB } & \multicolumn{2}{|c|}{ Tool } & \multicolumn{2}{|c|}{ Exp. } & \multicolumn{2}{|c|}{ Tool $\times$ CB } & \multicolumn{2}{|c|}{ CB $\times$ Exp. } & \multicolumn{2}{|c|}{ Tool $\times$ Exp. } & \multicolumn{2}{|c|}{ Tool $\times$ CB $\times$ Exp. } \\
\hline & $F_{1,24}$ & $p$-value & $F_{1,24}$ & $p$-value & $F_{1,24}$ & $p$-value & $F_{1,24}$ & $p$-value & $F_{1,24}$ & $p$-value & $F_{1,24}$ & $p$-value & $F_{1,24}$ & $p$-value \\
\hline Q1 & 1.00 & $=0.33$ & 0.94 & $=0.34$ & 1.94 & $=0.18$ & 2.35 & $=0.14$ & 0.55 & $=0.47$ & 9.10 & $<0.05$ & 0.06 & $=0.81$ \\
\hline Q2 & 0.91 & $=0.35$ & 7.98 & $<0.05$ & 7.81 & $<0.05$ & 2.45 & $=0.13$ & 0.61 & $=0.44$ & 0.00 & $=0.98$ & 0.00 & $=0.98$ \\
\hline Q3 & 0.02 & $=0.89$ & 14.2 & $<0.05$ & 2.35 & $=0.14$ & 0.70 & $=0.41$ & 0.57 & $=0.46$ & 0.04 & $=0.84$ & 0.08 & $=0.77$ \\
\hline Q4 & 0.10 & $=0.75$ & 2.10 & $=0.16$ & 8.18 & $<0.05$ & 0.34 & $=0.56$ & 0.12 & $=0.74$ & 0.81 & $=0.38$ & 0.38 & $=0.54$ \\
\hline Q5 & 0.97 & $=0.33$ & 0.16 & $=0.69$ & 1.07 & $=0.31$ & 4.44 & $<0.05$ & 0.02 & $=0.90$ & 0.23 & $=0.64$ & 0.16 & $=0.69$ \\
\hline Q6 & 0.38 & $=0.54$ & 0.28 & $=0.60$ & 3.35 & $=0.08$ & 0.14 & $=0.71$ & 0.00 & $=0.97$ & 0.09 & $=0.77$ & 0.07 & $=0.80$ \\
\hline Q7 & 0.09 & $=0.77$ & 1.10 & $=0.30$ & 11.3 & $<0.05$ & 0.43 & $=0.52$ & 0.05 & $=0.82$ & 0.10 & $=0.75$ & 0.28 & $=0.60$ \\
\hline Q8 & 0.11 & $=0.74$ & 0.27 & $=0.61$ & 3.04 & $=0.09$ & 0.27 & $=0.61$ & 0.67 & $=0.42$ & 0.03 & $=0.86$ & 0.00 & $=0.95$ \\
\hline
\end{tabular}

TABLE VIII: Chi-squared analysis on the post-study responses. Statistically significant results (95\% confidence) are highlighted. The numbers in VS and CP columns represent the total times each tool was selected by the participant in response to a question. The winner in each significant category is highlighted.

\begin{tabular}{cccll}
\hline Question & VS & CP & \multicolumn{3}{c}{ Chi-squared Test } \\
\hline SQ1 & 21 & 7 & $\chi^{2}(1, N=28)=7.00$ & $p<0.05$ \\
SQ2 & 8 & 20 & $\chi^{2}(1, N=28)=5.14$ & $p<0.05$ \\
SQ3 & 16 & 12 & $\chi^{2}(1, N=28)=0.57$ & $p=0.45$ \\
SQ4 & 17 & 11 & $\chi^{2}(1, N=28)=1.29$ & $p=0.26$ \\
SQ5 & 0 & 28 & $\chi^{2}(1, N=28)=28.00$ & $p<0.05$ \\
SQ6 & 11 & 13 & $\chi^{2}(1, N=24)=0.17$ & $p=0.68$ \\
SQ7 & 5 & 23 & $\chi^{2}(1, N=28)=11.57$ & $p<0.05$ \\
SQ8 & 7 & 21 & $\chi^{2}(1, N=28)=7.00$ & $p<0.05$ \\
SQ9 & 19 & 9 & $\chi^{2}(1, N=28)=3.57$ & $p=0.06$ \\
SQ10 & 15 & 13 & $\chi^{2}(1, N=28)=0.14$ & $p=0.71$ \\
SQ11 & 19 & 9 & $\chi^{2}(1, N=28)=3.57$ & $p=0.06$ \\
SQ12 & 16 & 11 & $\chi^{2}(1, N=27)=0.93$ & $p=0.34$ \\
\hline
\end{tabular}

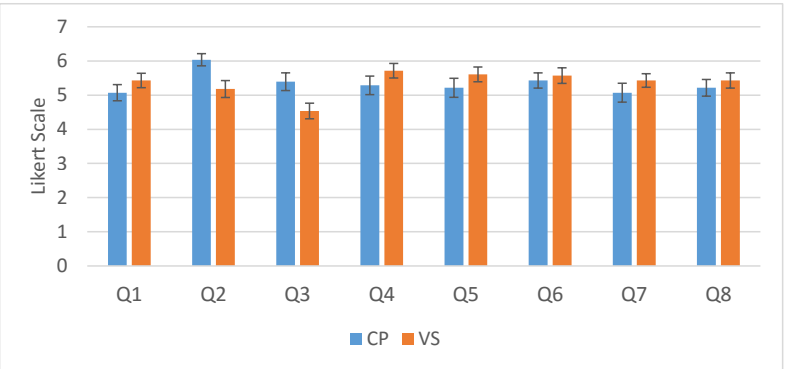

Fig. 4: Mean responses to the post-study questionnaire for $\mathrm{CP}$ and VS.

a decrease in the responses for VS. One possible explanation for the small superiority of $\mathrm{CP}$ over to VS on MG is that MG contains more code with larger classes (see Table I]. It could be that this larger size and the existence of more clutter give $\mathrm{CP}$ a slight edge when finding the definition of variables. This could potentially lead to the conclusion that $\mathrm{CP}$ is a more favorable tool for larger projects. However, this conclusion is 


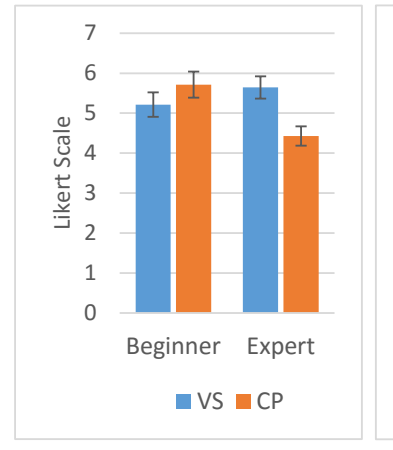

(a) Q1

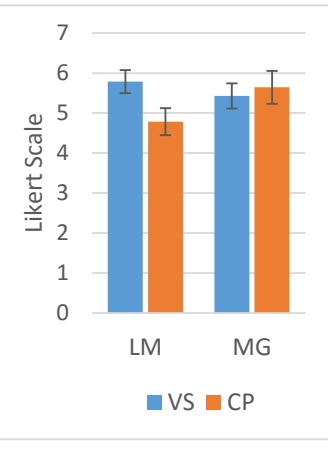

(b) Q5
Fig. 5: (a) Difference of averages between VS and CP grouped by user experience for Q1 responses. (b) Difference of averages between VS and CP grouped by codebase for Q5 responses.

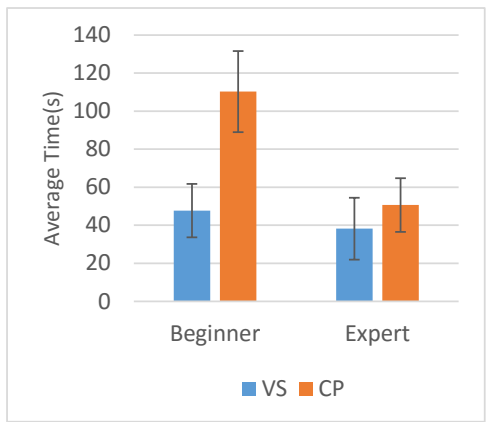

Fig. 6: The average time spent on completing T3 based on each tool and the participants' experience level.

premature and we believe a more detailed study is warranted to examine this interaction in more details.

On the tool level and from a quantitative point of view, it is evident that the choice of tool had a significant effect on time to completion of three tasks. Finding a valid username to login into the program (T1), determining the relationship between two classes (T3) and pinpointing a reasonable location in the code for adding the necessary logic to support some feature (T5). In all these cases, participants took significantly less time to complete their tasks with VS compared to CP. There are several possible explanations for this observations.

The first is the existence of transition animations in CP. As discussed in User Interface Design the animations were necessary to preserve the user's sense of environmental awareness. At any instance of time, the length of these animations depend on the camera's relative position to each code room and also the size of the codebase. Nevertheless, every animation was at least 1.5 seconds long. Considering a hypothetical situation where a user is in bird's view and wants to switch to firstperson mode to inspect a code room and then go back to bird's view, the transition animations take about 3 seconds. It takes a significantly less amount of time to open two files consecutively in VS.

Another possible explanation for observing faster task completion times with VS is that, $\mathrm{CP}$ provides more interaction capabilities compared to VS and 3D interactions are inherently slower compared to 2D interactions because of the added degree of freedom. When a user is inside a code room in $\mathrm{CP}$, they have the freedom of walking around, looking at the wallpapers or clicking on the wallpapers to inspect the code more closely. All these interactions take time. We also gained some insight about this issue from a different perspective. Often times, after we assigned a task to a participant, we noticed that some participants started to "play" with the interface or wander about aimlessly for a few seconds: due to the game-like environment of $\mathrm{CP}$, they occasionally walked around the room and inspected the visual aspects of the environment, or would make a verbal note about something in the environment that was irrelevant to the assigned task (such as how realistic the reflection effect was on the floor tiles, or how the grass texture looked unrealistic).

Focusing on the quantitative results based on the experience level of the participants, reveals other findings. As one would expect, and regardless of the tool, beginners generally took more time to finish their tasks compared to experts. The other result of interest is the observed interaction effect between the tool and the experience of the participants. Figure 6 presents the average time spent on determining the relationship between two classes (T3) based on each tool and the participants' experience level. The increase in the average time spent by the beginners when they switched from VS to CP was much more than this increase for experts. Without a more detailed study, it is difficult to draw any concrete conclusions. Informally, we think a possible explanation could be that it takes more time for the beginners to realize they do not know how to tackle T3. As a result, they take their time and explore the code further with $\mathrm{CP}$, hoping to find a clue that aids them in completing the task.

Considering the codebase aspect of our study and focusing on the quantitative results, we see that the choice of codebase only affected the time spent for completing T3 (determining the relationship between two classes) and T5 (pinpointing a reasonable location in the code for adding the necessary logic to support some feature). This observation can be explained by noting the difference in the sizes of the two codebases. As detailed in Table I. MG is larger and more cluttered than LM. For T5, where the participants needed to study the code more closely, it generally took them longer to browse through MG compared to LM. Other than task completion time, the choice of codebase did not significantly affect the qualitative results. This bolsters our initial assumption that the two codebases were very similar and the choice of the codebase, would not significantly affect our results.

\section{Evaluation: Understanding Project ORGANIZATION}

With the goal of determining the suitability of $\mathrm{CP}$ in the task of organizing an existing project, we designed and performed a second user study. In this user study, the users are tasked with organizing an existing project in Code Park in any way they saw fit. 


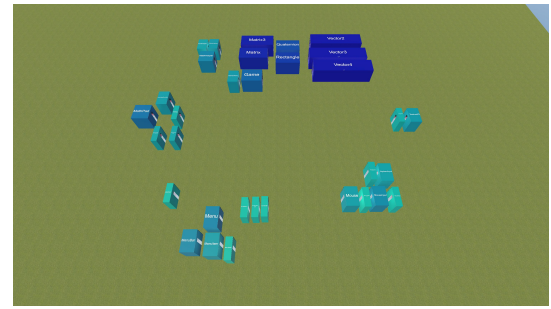

(a) Participant 1

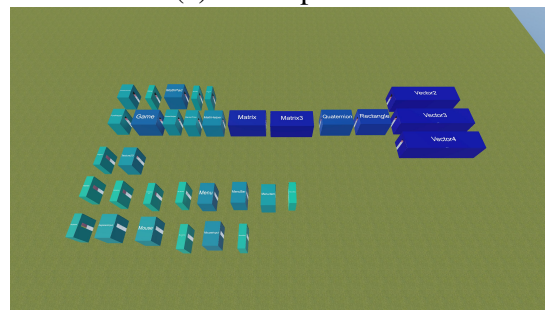

(d) Participant 4

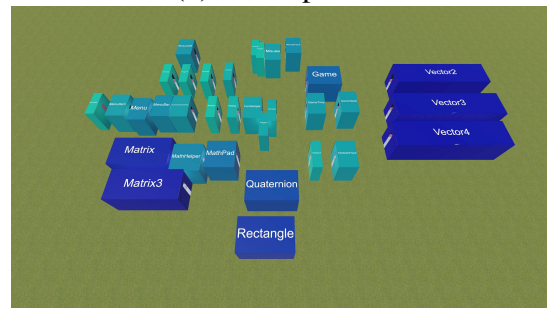

(g) Participant 7

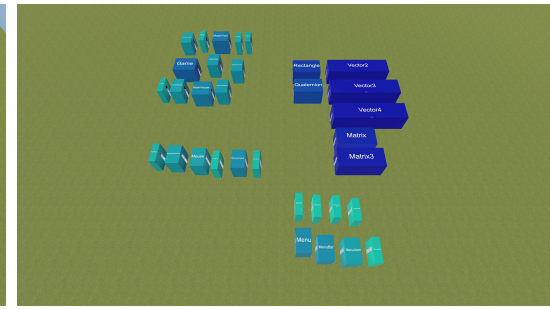

(b) Participant 2

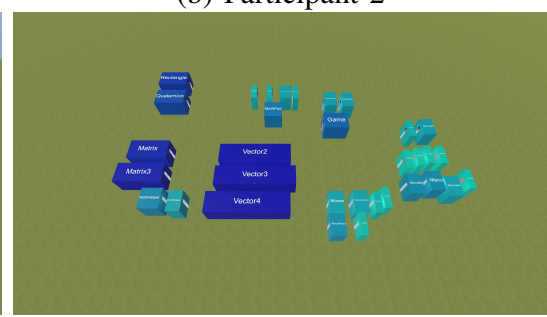

(e) Participant 5

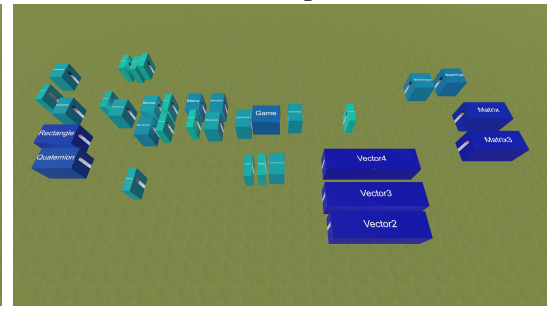

(h) Participant 8

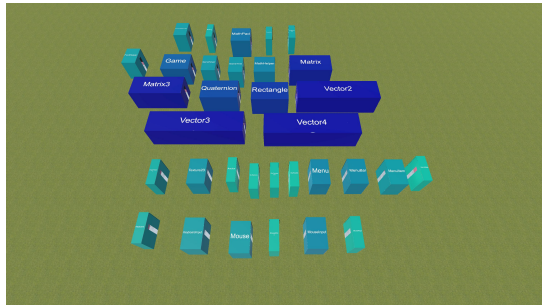

(c) Participant 3

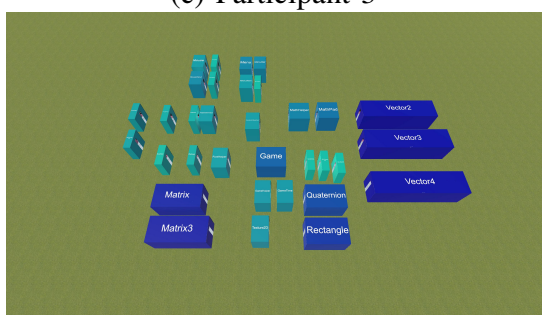

(f) Participant 6

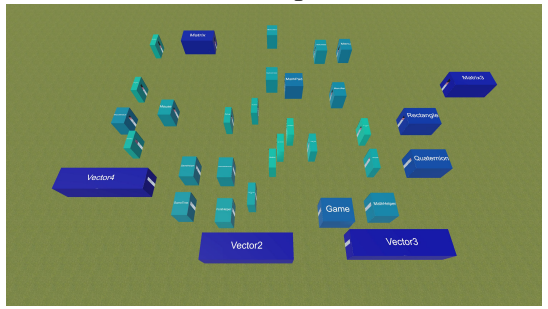

(i) Participant 9

Fig. 7: Screenshots of participants project organization.

Participants and Experience Design. We recruited 9 participants (8 males and 1 female ranging in age from 18 to 29 with a mean age of 22.8). Our requirements for participants were similar to the previous study, i.e. familiarity with the C\# language. Each participant was compensated with $\$ 10$ for their efforts at the end of the study session. Once again, each participant was given a pre-questionnaire containing some demographic questions as well as some questions asking about their experience in developing C\# applications.

Each participant was tasked with placing all 33 classes of an existing project in CP's environment in a manner that they saw reasonable. As a result, they were free to organize the classes in any way they preferred. We separated our participants into two groups of 5 and 4.

The classes in the project that was given to the first group were already organized into directories based on their relation (e.g. classes that handled user input were all inside of a directory called "input"). Conversely, the classes in the project that was given to the second group were not organized in any particular manner (i.e. all classes were inside the same directory). The goal of such separation was to observe whether grouping the classes based on their inherent relationships would affect users' decisions.

\section{A. Results}

As evident in Figure 7a to Figure 7e, the organization performed by the participants mostly followed the directory- based organization of the project that was given to them. Some participants chose to place the contents of each directory in a separate line while others chose to spatially group them into a group of adjacent blocks. We asked the participants about their reasoning for such arrangements and obtained the following responses:

Participant 1: "Folders were arranged spatially in groups. Classes that appeared related by name were sub-grouped."

Participant 2: "[I kept] directories grouped together."

Participant 3: "I just arranged classes of a particular folder in each row."

Participant 4: "The classes were arranged alphabetically for each folder and I arranged the classes in the same folder in the same line."

Participant 5: "I tried to group the related class together based on the usefulness and field."

Figure $7 \mathrm{f}$ to $7 \mathrm{i}$ depicts the results obtained from the second group of participants. When asked about their reasoning for their decisions, the following responses were obtained:

Participant 6: "When arranging the classes my first concern was to group similar classes together. After considering which groups existed, I tried to come up with a hierarchy based on the classes size. So I put 
bigger classes on the side and all the smaller ones in the middle."

Participant 7: "I tried to place the rooms in the chunk of similar classes. My priority was to place them in such a way that they are easy to find again." Participant 8: "I grouped the rooms based on their classes' name.”

Participant 9: "Big models together. Smaller ones in the middle so I can find them easier."

\section{B. Discussion}

In this user study our goal was to determine how users organize a project in $\mathrm{CP}$ environment in a way that the final result helped them remember the location of each class. The results show a possible relation between the user's cognitive understanding of the codebase and their decisions in organizing building block of the project when working with CP. As shown in Figure 7, users mostly chose to organize the constituent parts of the project based on their relationship with respect to each other.

In cases where the project files were already organized into directories, users mostly followed that same organization when working with CP. However, if the project lacked an inherent organization, users' decisions were guided either by the size of each class or the semantic relationship of those classes. Users mostly elected to organize similar parts of the project in the close proximity of each other. This is inline with the results observed in [39] where the increase in spatial dispersion of objects resulted in more difficulty in processing and attentional allocation.

\section{LimitATIONS AND FUTURE WORK}

There are a few notable limitations associated with the design of our first study and CP in general. First, we realize that our comparison with VS could potentially bias the results. This is because most C\# developers have experience with VS and such prior familiarity could affect their responses. Second, comparing VS which is fast and responsive to an interface that has animations and is slower may not result in a completely fair assessment.

Another limitation is that we compared VS against $\mathrm{CP}$ on a strict code understanding basis. Compared to VS, CP misses code editing or debugging functionalities. Also, CP currently only supports C\# codebases. However, it can be easily extended to any object-oriented programming language. Given these limitations, our results and the participants' feedback indicate a trend in preference for $\mathrm{CP}$ and tools that drastically change the way a programmer interacts with the code. Our results show that CP is at least as good as a professional tool such as VS in learning a codebase.

We plan to address these limitations by incorporate more functionality into $\mathrm{CP}$ such as a code editor and a debugger. These were the most requested features by our user study participants. We also would like to perform a more in-depth study to observe how $\mathrm{CP}$ would affect the learning of a group of novice programmers in a semester long course similar to the work of Saito et al. [37]. Zorn et al. [40] examined the game
Minecraft as a means of increasing interest in programming. It would be interesting to perform a similar study on $\mathrm{CP}$ and evaluate its effectiveness on programming.

Beyond system hardware limits, we believe CP can scale, through extended metaphors. Since a world of buildings will likely become incomprehensible, we expect a project to grow from buildings to districts, to cities, to regions, and so on. Understanding the boundary and limit of each is future work We also plan to evaluate $\mathrm{CP}$ in virtual reality (VR) and augmented reality (AR) environments. It would be interesting to design an $\mathrm{AR}$ system that employs $\mathrm{CP}$ to aid in code understanding while allowing programmers to naturally use the mouse and the keyboard.

\section{CONCLUSION}

We presented Code Park, a 3D code visualization tool designed to improve learning a codebase. Code Park lays out an existing codebase in a 3D environment and allows users to explore and study the code in two modalities, a bird's eye view mode and a first-person view mode. We performed a user study to evaluate the usability and the effectiveness of Code Park in comparison to Microsoft Visual Studio in performing a series of task related to the user's understanding of the codebase. We then performed a follow up user study to evaluate how users would organize an existing project in the 3D environment in a manner that would help them remember the codebase later. The analysis of our results demonstrated the benefits of Code Park as a viable tool for understanding an existing codebase. Our participants found Code Park to be easy to learn, instrumental in understanding as well as remembering the structure of a codebase, and enjoyable to use. Our analysis of the first study revealed that the participants that did not have a strong programming background found Code Park to be easier to work with compared to Microsoft Visual Studio. The result of second study showed that the users tend to organize project in a semantically meaningful form.

\section{ACKNOWLEDGEMENTS}

This work is supported in part by NSF Award IIS-1638060, Lockheed Martin, Office of Naval Research Award ONRBAA15001, Army RDECOM Award W911QX13C0052, and Coda Enterprises, LLC. We also thank the ISUE lab members at UCF for their support as well as the anonymous reviewers for their helpful feedback.

\section{REFERENCES}

[1] S. C. Eick, J. L. Steffen, and E. E. Sumner, "Seesoft-a tool for visualizing line oriented software statistics," IEEE Transactions on Software Engineering, vol. 18, no. 11, pp. 957-968, Nov 1992.

[2] A. Bragdon, S. P. Reiss, R. Zeleznik, S. Karumuri, W. Cheung, J. Kaplan, C. Coleman, F. Adeputra, and J. J. LaViola, Jr., "Code bubbles: Rethinking the user interface paradigm of integrated development environments," in Proceedings of the 32Nd ACM/IEEE International Conference on Software Engineering - Volume 1, ser. ICSE '10. New York, NY, USA: ACM, 2010, pp. 455-464.

[3] R. Wettel and M. Lanza, "Visually localizing design problems with disharmony maps," in Proceedings of the 4th ACM Symposium on Software Visualization, ser. SoftVis '08. New York, NY, USA: ACM, 2008, pp. 155-164. 
[4] A. D. Baddeley, Human memory: Theory and practice. Psychology Press, 1997.

[5] J. Sweller, "Cognitive load theory, learning difficulty, and instructional design," Learning and Instruction, vol. 4, no. 4, pp. 295 - 312, 1994.

[6] R. M. Carini, G. D. Kuh, and S. P. Klein, "Student engagement and student learning: Testing the linkages*," Research in Higher Education, vol. 47 , no. 1, pp. 1-32, 2006.

[7] N. Burgess, E. A. Maguire, and J. O'Keefe, "The human hippocampus and spatial and episodic memory," Neuron, vol. 35, no. 4, pp. 625 - 641, 2002.

[8] M. Balzer and O. Deussen, "Level-of-detail visualization of clustered graph layouts," in Visualization, 2007. APVIS '07. 2007 6th International Asia-Pacific Symposium on, Feb 2007, pp. 133-140.

[9] D. Bonyuet, M. Ma, and K. Jaffrey, "3d visualization for software development," in Web Services, 2004. Proceedings. IEEE International Conference on, July 2004, pp. 708-715.

[10] N. Hawes, S. Marshall, and C. Anslow, "Codesurveyor: Mapping largescale software to aid in code comprehension," in 2015 IEEE 3rd Working Conference on Software Visualization (VISSOFT), Sept 2015, pp. 96-105.

[11] Y. Tymchuk, L. Merino, M. Ghafari, and O. Nierstrasz, "Walls, pillars and beams: A 3d decomposition of quality anomalies," in 2016 IEEE Working Conference on Software Visualization (VISSOFT), Oct 2016, pp. 126-135.

[12] O. Greevy, M. Lanza, and C. Wysseier, "Visualizing feature interaction in 3-d," in 3rd IEEE International Workshop on Visualizing Software for Understanding and Analysis, 2005, pp. 1-6.

[13] G. Balogh and A. Beszedes, "Codemetrpolis: A minecraft based collaboration tool for developers," in 2013 First IEEE Working Conference on Software Visualization (VISSOFT), Sept 2013, pp. 1-4.

[14] L. Merino, M. Ghafari, O. Nierstrasz, A. Bergel, and J. Kubelka, "Metavis: Exploring actionable visualization," in 2016 IEEE Working Conference on Software Visualization (VISSOFT), Oct 2016, pp. 151-155.

[15] A. R. Teyseyre and M. R. Campo, "An overview of 3d software visualization," IEEE Transactions on Visualization and Computer Graphics, vol. 15 , no. 1, pp. 87-105, Jan 2009.

[16] P. Caserta and O. Zendra, "Visualization of the static aspects of software: A survey," IEEE Transactions on Visualization and Computer Graphics, vol. 17, no. 7, pp. 913-933, July 2011.

[17] A. Marcus, L. Feng, and J. I. Maletic, " $3 \mathrm{~d}$ representations for software visualization," in Proceedings of the 2003 ACM Symposium on Software Visualization, ser. SoftVis '03. New York, NY, USA: ACM, 2003, pp. 27-ff.

[18] C. Kurtz, "Code gestalt: A software visualization tool for human beings," in CHI '11 Extended Abstracts on Human Factors in Computing Systems, ser. CHI EA '11. New York, NY, USA: ACM, 2011, pp. 929-934.

[19] M. Lanza and S. Ducasse, "A categorization of classes based on the visualization of their internal structure: The class blueprint," SIGPLAN Not., vol. 36, no. 11, pp. 300-311, Oct. 2001.

[20] C. Gutwenger, M. Jünger, K. Klein, J. Kupke, S. Leipert, and P. Mutzel, "A new approach for visualizing uml class diagrams," in Proceedings of the 2003 ACM Symposium on Software Visualization, ser. SoftVis '03. New York, NY, USA: ACM, 2003, pp. 179-188.

[21] M. Balzer, O. Deussen, and C. Lewerentz, "Voronoi treemaps for the visualization of software metrics," in Proceedings of the 2005 ACM Symposium on Software Visualization, ser. SoftVis '05. New York, NY, USA: ACM, 2005, pp. 165-172.

[22] D. Holten, "Hierarchical edge bundles: Visualization of adjacency relations in hierarchical data," IEEE Transactions on Visualization and Computer Graphics, vol. 12, no. 5, pp. 741-748, Sept 2006.

[23] A. Cockburn and B. McKenzie, "Evaluating the effectiveness of spatial memory in $2 \mathrm{~d}$ and $3 \mathrm{~d}$ physical and virtual environments," in Proceedings of the SIGCHI Conference on Human Factors in Computing Systems, ser. CHI '02. New York, NY, USA: ACM, 2002, pp. 203-210.

[24] G. Robertson, M. Czerwinski, K. Larson, D. C. Robbins, D. Thiel, and M. van Dantzich, "Data mountain: Using spatial memory for document management," in Proceedings of the 11th Annual ACM Symposium on User Interface Software and Technology, ser. UIST '98. New York, NY, USA: ACM, 1998, pp. 153-162.

[25] H. Graham, H. Y. Yang, and R. Berrigan, "A solar system metaphor for 3d visualisation of object oriented software metrics," in Proceedings of the 2004 Australasian Symposium on Information Visualisation - Volume 35, ser. APVis '04. Darlinghurst, Australia, Australia: Australian Computer Society, Inc., 2004, pp. 53-59.

[26] M. Balzer, A. Noack, O. Deussen, and C. Lewerentz, "Software landscapes : Visualizing the structure of large software systems," in
Joint Eurographics - IEEE TCVG Symposium on Visualization (VisSym), 2004.

[27] T. Panas, T. Epperly, D. Quinlan, A. Saebjornsen, and R. Vuduc, "Communicating software architecture using a unified single-view visualization," in 12th IEEE International Conference on Engineering Complex Computer Systems (ICECCS 2007), July 2007, pp. 217-228.

[28] S. Alam and P. Dugerdil, "Evospaces visualization tool: Exploring software architecture in 3d," in 14th Working Conference on Reverse Engineering (WCRE 2007), Oct 2007, pp. 269-270.

[29] C. Knight and M. Munro, "Virtual but visible software," in Information Visualization, 2000. Proceedings. IEEE International Conference on, 2000, pp. 198-205.

[30] G. Langelier, H. Sahraoui, and P. Poulin, "Visualization-based analysis of quality for large-scale software systems," in Proceedings of the 20th IEEE/ACM International Conference on Automated Software Engineering, ser. ASE '05. New York, NY, USA: ACM, 2005, pp. 214-223.

[31] S. Cooper, W. Dann, and R. Pausch, "Alice: A 3-d tool for introductory programming concepts," in Proceedings of the Fifth Annual CCSC Northeastern Conference on The Journal of Computing in Small Colleges, ser. CCSC '00. USA: Consortium for Computing Sciences in Colleges, 2000 , pp. $107-116$.

[32] M. Resnick, J. Maloney, A. Monroy-Hernández, N. Rusk, E. Eastmond, K. Brennan, A. Millner, E. Rosenbaum, J. Silver, B. Silverman, and Y. Kafai, "Scratch: Programming for all," Commun. ACM, vol. 52, no. 11, pp. 60-67, Nov. 2009.

[33] D. Parsons and P. Haden, "Parson's programming puzzles: A fun and effective learning tool for first programming courses," in Proceedings of the 8th Australasian Conference on Computing Education - Volume 52, ser. ACE '06. Darlinghurst, Australia, Australia: Australian Computer Society, Inc., 2006, pp. 157-163.

[34] K. Kahn, "Toontalk tman animated programming environment for children," Journal of Visual Languages \& Computing, vol. 7, no. 2, pp. 197-217, 1996.

[35] E. Perrin, S. Linck, and F. Danesi, "Algopath: A new way of learning algorithmic," in in The Fifth International Conference on Advances in Computer-Human Interactions, 2012.

[36] C. Kelleher and R. Pausch, "Using storytelling to motivate programming," Commun. ACM, vol. 50, no. 7, pp. 58-64, Jul. 2007.

[37] D. Saito, H. Washizaki, and Y. Fukazawa, "Influence of the programming environment on programming education," in Proceedings of the 2016 ACM Conference on Innovation and Technology in Computer Science Education, ser. ITiCSE '16. New York, NY, USA: ACM, 2016, pp. 354-354.

[38] J. O. Wobbrock, L. Findlater, D. Gergle, and J. J. Higgins, "The aligned rank transform for nonparametric factorial analyses using only anova procedures," in Proceedings of the SIGCHI Conference on Human Factors in Computing Systems, ser. CHI '11. New York, NY, USA: ACM, 2011, pp. 143-146.

[39] A. B. Abbes, E. Gavault, and T. Ripoll, "The effect of spatial organization of targets and distractors on the capacity to selectively memorize objects in visual short-term memory," Advances in cognitive psychology, vol. 10, no. 3, p. 90, 2014.

[40] C. Zorn, C. A. Wingrave, E. Charbonneau, and J. J. LaViola Jr, "Exploring minecraft as a conduit for increasing interest in programming," in Proceedings of the International Conference on the Foundations of Digital Games 2013 (FDG 2013), 2013, pp. 352-359. 